\title{
O CONSUMO DE ÁLCOOL EM UNIVERSITÁRIOS: FIDEDIGNIDADE E SENSIBILIDADE DE UMA ESCALA DE MEDIDA
}

\section{Nilton Formiga}

Faculdade Maurício de Nassau.

\begin{abstract}
Resumo
O consumo de álcool entre os jovens tem sido não somente um determinante para as relações interpessoais, mas, também, é atualmente, um problema que traz um prejuízo social, econômico e de saúde. Devido a essa condição, têm surgido instrumentos e reavaliados outras medidas mais confiáveis para identificar o problema com o consumo de álcool nesse grupo; dos inúmeros instrumentos, o AUDIT tem sido o mais utilizado em estudos no mundo, o qual tem se revelado consistente. O presente estudo tem como objetivo, avaliar a fidedignidade e consistência interna do AUDIT a partir de distintos cálculos estatísticos em uma amostra de universitários. Duas amostras de universitários, homens e mulheres, de 18 a 40 anos, na cidade de João Pessoa-PB responderam o AUDIT e dados sócio-demográficos. A partir de diferentes cálculos estatísticos, observaram-se indicadores psicométricos que revelaram confiabilidade e fidedignidade do AUDIT nas referidas amostras, garantindo assim, a medida sobre a identificação do problema do consumo de álcool nos respondentes.
\end{abstract}

Palavras-chave: problemas com álcool; fidedignidade; universitários.

\section{THE ALCOHOL CONSUMPTION IN UNIVERSITY STUDENTS: RELIABILITY AND SENSITIVITY OF A SCALE OF MEASUREMENT}

\begin{abstract}
Alcohol consumption among young people has been not only a key to interpersonal relationships, but also is currently an issue that brings social issues such as economic and health damage. Due to this condition, there have been created and suggested many instruments and also search for revaluation in many of the instruments to test their reliability measure for identifying the issue with alcohol consumption in this group; from countless instruments, the AUDIT has been the most used in studies around the world, which has proved consistent. The present study aims to take measure on the reliability and internal consistency of the AUDIT from different statistical calculations in a sample taken from university students. Two samples of college students, both men and women, from ages 18-40 years old, in the city of João Pessoa -PB, answered the AUDIT and also a socio-demographic data. Performing different statistical calculations, it has been observed psychometric indicators, which revealed reliability and trustworthiness of the AUDIT in the samples tested, thus ensuring the measure on the identification of an alcohol consumption issue in the respondents.
\end{abstract}

Keywords: alcohol issues; reliability; university students. 


\title{
CONSUMO DE ALCOHOL EN UNIVERSITARIO: FIABILIDAD Y SENSIBILIDAD DE UNA ESCALA PARA MEDIR
}

\begin{abstract}
Resumen
El consumo de alcohol entre los jóvenes no sólo ha sido un factor clave en las relaciones interpersonales, sino que es actualmente un tema que trae un daño social, económico y sanitario. Debido a esta condición, ha habido instrumentos reevaluados y otras medidas más fiables para la identificación del problema con el consumo de alcohol en jovenes; de muchos instrumentos, o AUDIT ha sido el más utilizado en estudios en el mundo sobre el alcohol, demostrado ser consistente. El presente estudio tiene como objetivo evaluar la fiabilidad y consistencia interna del cuestionario AUDIT en diferentes estadísticas en una muestra de universitario. Dos muestras de hombres y mujeres de la universidad, 18 a 40 años de edad, de la ciudad de João Pessoa-PB contestaron el AUDIT y datos socio- demográficos. Los diferentes cálculos estadísticos revelaron indicadores psicométricos fiabilidad del AUDIT en estas muestras, asegurando así la medida en la identificación del problema del consumo de alcohol en los encuestados.
\end{abstract}

Palabras clave: problemas con el alcohol; fiabilidad; universidad.

\section{INTRODUÇÃO}

Historicamente, apesar de encontrar relatos quanto a ingestão de álcool por parte do ser humano ter ocorrido há tempos e não ser um fenômeno, exclusivo, da contemporaneidade; é também relatado que tal condição, na dinâmica interpessoal, é sempre justificada como critério, sine quo non, para a realização de comemorações publicas ou privadas, relações íntimas (namoro, casamento, etc.) e até sugerido como fator de proteção e redução de doenças cardiovasculares. Mesmo que uma perspectiva positiva quanto ao uso moderado da ingestão de álcool, ainda é destaque o amplo envolvimento desse fenômeno em problemas sociais, econômicos e de saúde (Garcia, Aguilar \& Facundo, 2008; Agante, 2009; Martins, 2009; Bye \& Rossow, 2010).

Atualmente, o problema sobre o uso abusivo de álcool vem recebendo uma atenção política e científica urgente; tal fato observa-se, seja na mídia em geral ou nos estudos especializados sobre o tema, que vem aumento o uso dessa substância por parte dos jovens e jovens-adultos e o que tem sido mais alarmante é o consumo ocorrido em nível etário mais baixo neste grupo. Os motivos são os mais diversos: influência dos pares de iguais, da cultura, da socialização familiar, dos envolvimentos comemorativos, entre outros contextos (Carlini-Contrim, Gazal-Carvalho \& Gouveia, 2000; Navarro \& Pontillo, 2002; Silva, Malbergier, Stempliuk \& Andrande, 2006; Carlini, Carlini-Contrim \& SilvaFilho, 2007; Kerr-Corrêa el al., 2001; Kerr-Corrêa et al., 2002; Agante, 2009; Fachini, 2009; Breda, 2010; Carvalho, 2010; Dallo \& Martins, 2011). 
Com isso, as áreas da ciência da saúde, humana e social têm avaliado o perfil dos consumidores do uso de álcool e a relação dessa substância na vida das pessoas, tendo como objetivo o mapeamento da motivação e a avaliação do impacto nas variáveis psicológicas (por exemplo, personalidade, dinâmica familiar, autoconceito, autoestima, etc.). Essa condição contribui para a compreensão da iniciação, constância e padrão de consumo dessa substância, até porque a condição no uso de álcool tem causado dano à saúde física e mental, prejuízo social e nas relações interpessoais, associando-se, em alguns momentos, às variáveis da delituosidade (por exemplo, condutas desviantes, comportamento agressivo, etc.) (Pastor, Balaguer \& García-Merita, 2000; Navarro \& Pontillo, 2002; Llorens, Palmer \& Perellón del Rio, 2005; Chalub \& Telles, 2006; Silva, Malbergier, Stempliuk \& Andrande, 2006; Musitu, Jiménez \& Murgui, 2007; Romera, 2008; Andrade, Anthony \& Silveira, 2009; Formiga, 2011).

Frente a tal situação é que se exigiu uma avaliação em relação à prevalência, freqüência e padrão do consumo de álcool; para isso, elaborou-se uma medida com objetivo de rastrear o consumo problemático e o padrão de ingestão dessa substância. Foi então que a OMS (World Health Organization, 2004), orientada pelos manuais diagnósticos CID - 10 e DSM - III (American Psychiatric Association, APA, 1994), organizou critérios em relação a dependência do uso de álcool, elaborando e estruturando, com isso, um dos instrumentos mais usados para avaliar o consumo excessivo do álcool; este instrumento é intitulado de Alcohol Use Disorders Identification Test (AUDIT) conhecido no Brasil como Teste de Identificação de Problemas Relacionados ao Uso de Álcool. Este teste foi desenvolvido por Babor, Higgins-Biddle, Saunders \& Monteiro (2001; Babor et al. 1992; Babor \& Higgins-Biddle, 2001), traduzido e validado por Figlie, Pillon, Laranjeira e Dunn (1997) no Brasil, o qual destinou-se à avaliação inicial ou rastreio sobre o consumo exagerado do álcool e outras drogas e, a partir dos resultados observados, propor intervenções sociais e psicológicas frente aos prejuízos do consumo dessa substância.

Para facilitar a avaliação do problema enfrentado pelo usuário de álcool, os pesquisadores sobre o tema utilizam um ponto de corte na distribuição dos escores dos respondentes; esses escores variam de zero (0) a 40 e podem ser conhecidos quando se soma as questões do instrumento, independente da dimensão do AUDIT. A distribuição desses escores destinados à avaliação do 
problema no consumo de álcool pode ser assim classificada em zonas: na zona I, indica uso de baixo risco ou abstinência ou educação no uso, a zona II, sugere uso de risco ou uso simples, a zona III sugere uso nocivo ou aconselhamento simples + intervenção breve + monitoramento continuado, por fim, a zona IV, demonstra possível dependência ou referencia o diagnóstico, avaliação e tratamento. (Babor, Higgins-Biddle, Saunders \& Monteiro, 2001; Pillon \& CorradiWebster, 2006).

Considerando a importância do AUDIT, vê-se a necessidade de avaliar a consistência, fidedignidade e sensibilidade deste instrumento em universitários em relação a mensuração, avaliação e diagnóstico do problema com o uso do álcool. Nos estudos sobre o AUDIT, não foi encontrado resultado que saliente diferentes análises estatísticas em um único estudo, enfatizando elementos teóricos, metodológicos e psicométricos de validação e confiabilidade (Babor et al., 1992; Figlie, et. all., 1997; Babbor, Higgins-Biddle, Saunders \& Monteiro, 2001; Pillon \& Corradi-Webster, 2006; Moretti-Pires \& Corradi-Webster, 2011).

\section{MÉTODO}

\section{Amostra}

Com objetivo de avaliar a segurança psicométrica da escala, em termos de teste-restes, duas amostras compuseram o estudo, uma com 201 sujeitos e outra com 237 sujeitos. Nestas, os sujeitos apresentavam idade de 18 a 40 anos ( $M=23,71$; d.p. $=4,99$ ), sendo $60 \%$ de mulheres, todos da cidade de João Pessoa-PB, distribuídos no nível de ensino superior de instituições privadas e publicas nos curso de ciência humana e da saúde (Administração, Direito, Educação Física, Enfermagem). A amostra foi não probabilística, pois se considerou o sujeito que, consultado, se dispôs a colaborar, respondendo o questionário a ele apresentado.

\section{Instrumentos}

Os sujeitos responderam um questionário com o seguinte instrumento: Teste de identificação de problemas relacionados ao uso de álcool - AUDIT. Trata-se de um questionário desenvolvido para Organização Mundial da Saúde, o qual foi organizado de acordo com Classificação Internacional de Doença (CID10) destinada ao rastreamento para uso problemático (especificamente, o uso nocivo e dependência) de álcool durante um período de 12 meses (Babor, Fuente, Saunders \& Grant, 1992; Babor \& Higgins-Biddle, 2001; Babor, Higgins- 
Biddle, Saunders \& Monteiro, 2001; Saunders, Aasland, Babor, Fuente \& Grant, 1993).

No Brasil, foi Figlie, Pillon, Laranjeira e Dunn (1997) que validaram o AUDIT com o objetivo de avaliar os problemas relacionados ao álcool em contexto hospitalar e em adolescentes, jovens, jovens adultos e adultos em diversos países, inclusive no Brasil (Bergman \& Källmén, 2002; Kerr-Corrêa, Dalben, Trinca et al., 2001; Kerr-Corrêa, Simão, Dalben et al., 2002); em todas essas amostras os instrumento revelou indicadores estatísticos confiáveis.

O instrumento AUDIT contém 10 questões em relação ao uso do álcool em relação ao último ano de consumo; de acordo Pillon e Corradi-Webster (2006) estas questões são organizadas fatorialmente em três dimensões: a primeira, refere-se a uma medida sobre a quantidade e a frequência do uso regular ou ocasional de álcool (por exemplo, freqüência de uso, quantidade em um dia típico e freqüência de beber pesado) categorizada como Padrão consumo de álcool; as três questões seguintes investigam sintomas de dependência (por exemplo, dificuldade de controlar o uso, aumento da importância da bebida e beber pela manhã) categorizadas como Sinais e sintomas de dependência; por fim, as quatro questões finais, referem-se a problemas recentes na vida relacionados ao consumo (por exemplo, sentimento de culpa após o uso de álcool, esquecimentos após o uso, lesões causadas pelo uso do álcool e preocupação de terceiros) e que são categorizadas como Problemas decorrentes do álcool.

De acordo com os autores supracitados, os escores variam de zero (0) a 40 e são obtidos por meio da somatória das questões do AUDIT. Uma pontuação igual ou superior a oito refere-se a um padrão de risco ou uso problemático de álcool, para pontuações inferiores a esse escore considera-se uso não problemático ou baixo risco. O AUDIT tem uma grande vantagem de permitir classificar em quatro padrões de uso ou nível de risco quanto ao consumo; estes níveis são organizados em Zonas.

O conceito de Zonas de Risco é interessante pelos seguintes motivos: pautando-se no conceito de prevenção do uso de álcool, permite distanciamento da visão baseada na dependência - que dicotomiza os pacientes em dependentes e não dependentes - instituindo padrões gradativos de uso; em decorrência, as Zonas Contínuas permitem um enfoque na prevenção, uma vez que o paciente é sensibilizado para a redução do uso de álcool, sendo estimulado para ingresso 
em Zona de menor risco (Moretti-Pires \& Corradi-Webster, 2011). Desta forma, as zonas avaliativas são as seguintes: zona I (Até 7 pontos: indica uso de baixo risco ou abstinência); zona II (de 8 a 15 pontos: sugere uso de risco); zona III (de 16 a 19 pontos: sugere uso nocivo) e zona IV (acima de 20 pontos: demonstra possível dependência) (Babor, et al., 2004; Moretti-Pires \& CorradiWebster, 2011).

\section{Procedimentos}

Todos os procedimentos adotados nesta pesquisa seguiram as orientações previstas na Resolução 466/2012 do CNS e na Resolução 016/2000 do Conselho Federal de Psicologia para as pesquisas com seres humanos (Conselho Nacional de Saúde [CNS], 2012; Associação Nacional de Pesquisa e Pós-Graduação em Psicologia [ANPEPP], 2000).

Administração

Colaboradores com experiência prévia na administração do instrumento foram responsabilizados pela coleta dos dados, e apresentaram-se as pessoas nas instituições de ensino superior, na cidade de João Pessoa-PB, como interessados em conhecer as opiniões e os comportamentos deles sobre as questões descritas no instrumento da pesquisa.

Solicitou-se a colaboração voluntária das pessoas no sentido de responderem um breve questionário. Após ficarem cientes das condições de participação na pesquisa, assinaram um termo de Consentimento Livre e Esclarecido. Foi-lhes dito que não havia resposta certa ou errada. A todos foi assegurado o anonimato das suas respostas informando que estas seriam tratadas em seu conjunto. A escala foi respondida individualmente, em sala de aula, logo após a autorização do coordenador do curso e professor da disciplina que seria ocupada para a administração da pesquisa.

Apesar de o instrumento ser autoaplicável, contando com as instruções necessárias para que as pessoas possam responder as questões exigidas nos instrumento, os colaboradores na aplicação estiveram presentes durante toda a administração do mesmo para retirar eventuais dúvidas ou realizar esclarecimentos que se fizessem indispensáveis. Um tempo médio de 30 minutos foi suficiente para concluir essa atividade.

Análise dos dados

Quanto à análise dos dados, realizou-se, além das análises descritivas e de correlação de Pearson, o calculo do alfa de Cronbach ( $\square$ ) e curva de ROC; além 
desses cálculos, realizou-se uma análise fatorial confirmatória a fim de avaliar a consistência estrutural do modelo já previamente encontrado. Considerou-se como entrada a matriz de covariâncias o estimador ML (Maximum Likelihood). Sendo um tipo de análise estatística mais criteriosa e rigorosa, buscou-se avaliar a estrutura teórica do AUDIT com três fatores. A seguir serão apresentados esses indicadores: $O X^{2}$ (qui-quadrado) testa a probabilidade do modelo teórico se ajustar aos dados: quanto maior o valor do $x^{2}$ pior o ajustamento. Entretanto, ele tem sido pouco empregado na literatura, sendo mais comum considerar sua razão em relação aos graus de liberdade $\left(\mathrm{x}^{2} / \mathrm{g} . \mathrm{l}\right.$.). Neste caso, valores até 3 indicam um ajustamento adequado; Raiz Quadrada Média Residual (RMR), que indica o ajustamento do modelo teórico aos dados, na medida em que a diferença entre os dois se aproxima de zero; O Goodness-of-Fit Index (GFI) e o Adjusted Goodness-of-Fit Index (AGFI) são análogos ao $\mathrm{R}^{2}$ na regressão múltipla e, portanto, indicam a proporção de variância-covariância nos dados explicada pelo modelo. Os valores desses indicadores variam de 0 a 1 , sendo que os valores na casa dos 0,80 e 0,90, ou superiores, indicam um ajustamento satisfatório; A Root-Mean-Square Error of Approximation (RMSEA), com seu intervalo de confiança de 90\% (IC90\%), é considerado um indicador de "maldade" de ajuste, isto é, valores altos indicam um modelo não ajustado. Assume-se como ideal que o RMSEA se situe entre 0,05 e 0,08, aceitando-se valores até 0,10; O Comparative Fit Index (CFI) - compara de forma geral o modelo estimado ao modelo nulo, considerando valores mais próximos de um como indicadores de ajustamento satisfatório; O Expected Cross-Validation Index (ECVI) e o Consistent Akaike Information Criterion (CAIC) são indicadores geralmente empregados para avaliar a adequação de um modelo determinado em relação a outro. Valores baixos do ECVI e CAIC expressam o modelo com melhor ajuste (Joreskög \& Sörbom, 1989; Kelloway, 1998; Hair, Anderson, Tatham \& Black, 2005).

\section{RESULTADOS E DISCUSSÃO}

A fim de atender o objetivo do presente estudo, efetuou, inicialmente, uma análise dos alfas de Cronbach ( $\alpha$ ) em várias secções de amostras (ver Tabela 1); este cálculo revelou a existência de alfas acima de 0,70 em todas as secções, condição que sugere a confiabilidade do referido instrumento, tanto ao tratá-lo de forma unifatorial (pontuação total dos itens, AUDIT total) quanto na sua 
distribuição fatorial (por exemplo, Padrão Consumo de Álcool, Sinais Sintoma de Dependência e Problemas Uso Álcool). Seja considerando a amostra total ou por temporalidade (primeira e segunda amostras) ou através de uma forma amostral alternativa, a qual se refere ao método das duas metades ou entre os grupos de idades (sujeitos mais novos e mais velhos), os resultados se mostraram confiáveis, garantindo assim, a fidedignidade da medida do uso excessivo de álcool. Vale destacar que, também, observou-se com tal método uma correlação de Pearson ( $r$ ) entre os fatores do AUDIT e a pontuação total entre as duas metades que variou de 0,25 a 0,32.

Tabela 1.

Indicadores alfas de Cronbach do AUDIT em distintas amostras.

\begin{tabular}{|c|c|c|c|c|c|c|c|}
\hline \multirow[b]{3}{*}{ Fatores do AUDIT } & \multicolumn{7}{|c|}{ Alfa de Cronbach $(\alpha)$} \\
\hline & \multirow[b]{2}{*}{$N_{\text {total }}$} & \multirow[b]{2}{*}{ N1 } & \multirow[b]{2}{*}{ N2 } & \multicolumn{2}{|c|}{$N_{\text {Duas metades }}$} & \multicolumn{2}{|c|}{$\mathrm{N}_{\text {Idade }}$} \\
\hline & & & & Metade 1 & Metade 2 & $18-22$ & $23-40$ \\
\hline PCA & 0,82 & 0,85 & 0,78 & 0,85 & 0,77 & 0,82 & 0,83 \\
\hline SSD & 0,88 & 0,77 & 0,75 & 0,79 & 0,75 & 0,86 & 0,90 \\
\hline PUA & 0,84 & 0,75 & 0,78 & 0,76 & 0,74 & 0,82 & 0,85 \\
\hline AUDIT $_{\text {total }}$ & 0,91 & 0,87 & 0,92 & 0,89 & 0,81 & 0,90 & 0,92 \\
\hline
\end{tabular}

Notas: $\mathrm{N}_{\text {total }}=$ amostra geral com 438 sujeitos; N1=primeira amostra com 201 sujeitos; N2=primeira amostra com 237 sujeitos; $N_{\text {duas metades }}=a m o s t r a$ total divida em duas metades; $\mathrm{N}_{\text {idade }}=$ grupo etário; $\mathrm{PCA}=$ Padrão Consumo de Álcool; $\mathrm{SSD}=$ Sinais Sintoma de Dependência; PUA=Problemas Uso Álcool.

Tendo os alfas, destacados acima, contribuído para corroborar a fidedignidade da medida do AUDIT em universitários, optou-se (justificando, como mais um elemento estatístico que possa garantir a confiança de medida neste instrumento) na referida amostra, avaliar, através da análise fatorial confirmatória, mais uma informação que venha contribuir para confirmação da consistência do AUDIT. Empregou-se, então, o pacote estatístico AMOS 21.0 para efetivara a análise fatorial confirmatória e testou-se o modelo da estrutura fatorial proposta pelos autores supracitados (por exemplo, Figlie, Pillon, Laranjeira \& Dunn, 1997; Pillon \& Corradi-Webster, 2006): deixando livre as covariâncias (phi, $\varphi$ ) entre os fatores, os resultados revelaram, além dos indicadores de qualidade de ajuste para o modelo trifatorial em diferentes seç̧ões amostrais, os quais se mostraram próximos as recomendações apresentadas na literatura (Byrne, 1989; Van De Vijver \& Leung, 1997) (ver Tabela 2). 
Tabela 2.

Indicadores psicométricos da estrutura fatorial do AUDIT.

\begin{tabular}{|c|c|c|c|c|c|c|c|c|c|c|}
\hline & & \multicolumn{9}{|c|}{ Indicadores psicométricos do AUDIT (três fatores*) } \\
\hline & & $\chi^{2} / g \mid$ & RMR & GFI & AGFI & CFI & TLI & RMSEA & CAIC & ECVI \\
\hline $\mathrm{N}_{\text {total }}$ & & 1,85 & 0,04 & 0,99 & 0,96 & 1,00 & 0,99 & 0,04 & 295,29 & 0,99 \\
\hline \multirow[t]{2}{*}{ Amostras } & N1 & 1,43 & 0,03 & 0,97 & 0,95 & 0,98 & 0,99 & 0,05 & 239,49 & 0,48 \\
\hline & N2 & 1,13 & 0,02 & 0,98 & 0,95 & 0,99 & 0,99 & 0,02 & 243,56 & 0,39 \\
\hline \multirow[t]{2}{*}{ Duas metades } & N3 & 1,44 & 0,05 & 0,97 & 0,94 & 0,99 & 0,98 & 0,04 & 217,67 & 0,43 \\
\hline & N4 & 1,71 & 0,05 & 0,97 & 0,92 & 0,99 & 0,97 & 0,05 & 287,16 & 0,51 \\
\hline \multirow[t]{2}{*}{ Idades } & N5 & 2,00 & 0,05 & 0,96 & 0,91 & 0,99 & 0,97 & 0,06 & 247,20 & 0,49 \\
\hline & N6 & 1,87 & 0,03 & 0,99 & 0,96 & 1,00 & 0,99 & 0,01 & 266,38 & 0,44 \\
\hline
\end{tabular}

Notas: $\mathrm{N}_{\text {total }}=$ amostra geral com 438 sujeitos; N1=primeira amostra com 201 sujeitos; N2=primeira amostra com 237sujeitos; Duas metades: N3=amostra primeira metade, N4=amostra segunda metade; Idades: N5=sujeitos de 18 a 21 anos e N6=sujeitos de 22 a 40 anos; *três fatores (PCA=Padrão Consumo de Álcool; SSD=Sinais Sintoma de Dependência; PUA=Problemas Uso Álcool).

Destaca-se que todas as saturações (Lambdas, $\lambda$ ) tanto estiveram dentro do intervalo esperado ( 0 a 1 ) quanto foram estatisticamente diferentes de zero $(t>1,96, p<0,05)$, denotando não haver problemas da estimação proposta da escala, o que corrobora a existência do modelo trifatorial hipotetizado. Com isso, observou-se uma associação lambda $(\lambda)$ positiva entre os fatores (Padrão Consumo de Álcool, Sinais Sintoma de Dependência e Problemas Uso Álcool), os quais variaram de 0,43 a 0,87. Desta forma, não apenas justifica a fidedignidade da estrutura fatorial do AUDIT, mas que o instrumento poderá ser administrado em universitários de forma confiável, independente da distribuição seccional.

Tendo garantido a consistência interna e estrutural do instrumento, buscouse, por fim, avaliar a sensibilidade e a especificidade do instrumento, tendo como orientação a quantificação e exatidão de um teste diagnóstico; para isso, recorreu-se à análise de Curva ROC, a qual tem por objetivo avaliar as especificidades do instrumento (Oliveira \& Andrade, 2002; Margoto, 2010) (ver Gráfico 1). Assim, pode-se destacar no gráfico abaixo, que a curva de ROC, por se encontrar acima do eixo, permite salientar uma garantia dessa medida na referida amostra. 


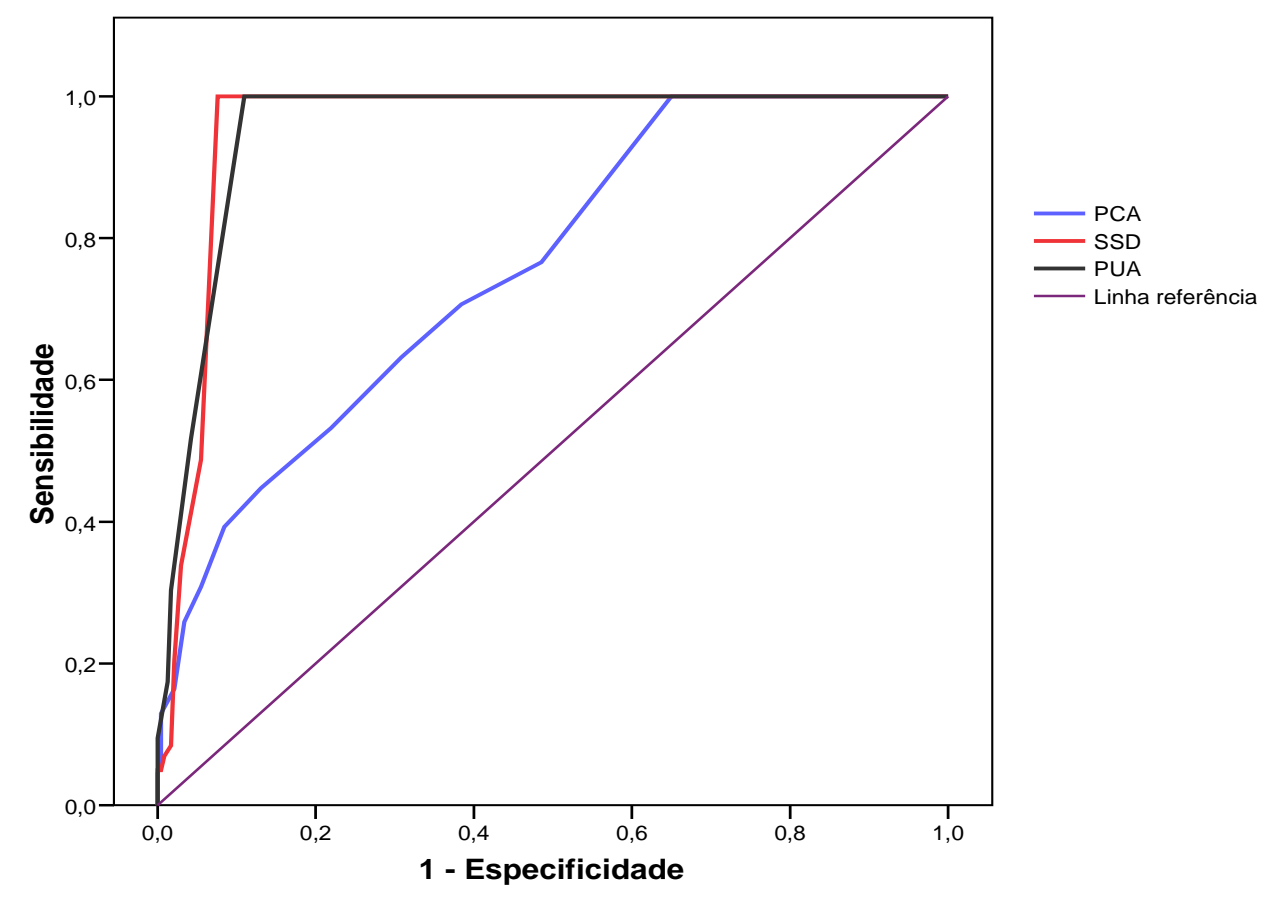

Figura 1.

Curva ROC em relação a sensitividade e especificidade da medida das dimensões do AUDIT.

Para tal foram calculadas as três dimensões do AUDIT na amostra, tendo encontrado uma área do ROC que variou de 0,77 a 0,96 (ver Tabela 3). Este resultado demonstra que as probabilidades previstas são verdadeiras e correspondem bem ao efeito real esperado (ver Gráfico1). Isto é, considerando a área de cobertura no gráfico 1 , bem como, os indicadores estatísticos expressos para a curva de ROC, o instrumento AUDIT é sensível para a identificação sobre problemas do consumo de álcool em universitários.

Tabela 3.

Indicadores estatísticos da área de ROC para as dimensões do AUDIT. IC95\%

\begin{tabular}{cccccc} 
& Área & Erro & $\mathrm{p}<$ & Limite inferior & Limite superior \\
\hline PCA & 0,77 & 0,02 & 0,01 & 0,71 & 0,80 \\
SSD & 0,95 & 0,01 & 0,01 & 0,93 & 0,98 \\
PUA & 0,96 & 0,01 & 0,01 & 0,93 & 0,97 \\
\hline
\end{tabular}

Notas: PCA=Padrão Consumo de Álcool; SSD=Sinais Sintoma de Dependência; PUA =Problemas Uso Álcool.

De forma geral, a partir desses resultados, destaca-se que o instrumento AUDIT é fidedigno e consistente, permitindo corroborar a estrutura trifatorial, a qual foi capaz de mensurar o problema do consumo com o álcool em 
universitários, revelando a consistência das dimensões - em medida psicométrica e interrelação - do padrão consumo de álcool, sinais sintoma de dependência e problemas uso álcool. As diversas análises realizadas para avaliação da escala apresentaram garantias psicrométricas e uma maior confiabilidade para tal medida, tendo por base diferentes evidências empíricas. Sendo assim, sugere aplicação e mensuração em outros contextos brasileiros com universitários, pois os indicadores comumente tidos em conta para corroborar o modelo estrutural proposto $\left(X^{2} / g l\right.$, GFI, AGFI, RMR, CFI, TLI, RMSEA), bem como os alfas de Cronbach e os estimadores da curva de ROC (ver Tabelas 1, 2 e 3) foram muito satisfatórios.

O presente estudo enfatizou uma medida para avaliar o consumo excessivo de álcool em universitários, para o qual é destacável, orientando-se pelos resultados aqui apresentados, a existência de fatores sobre o fenômeno da ingestão de álcool para este grupo; essa condição sugere ao tema em questão, não apenas a inserção em um grave problema social e psicológico, capaz de influenciar no desenvolvimento emocional e comportamental do sujeito universitário (Formiga, Picanço, Santos \& Souza, 2013), mas, também, a necessidade de tomar quanto orientação de políticas públicas educacionais e de intervenção a necessidade de um indicador de saúde mental para a análise do desenvolvimento psicossocial dos sujeitos pesquisados e a prevenção do excesso e dependência no uso de álcool.

Desta forma, pretende-se com tal estudo apresentar índices psicométricos no que se refere a avaliação do problema do uso álcool no contexto social e educacional de universitários; ao considerar os achados do estudo, deve-se salientar a urgência, devido a sua gravidade na vida dos jovens e jovens-adultos (Lucas, et al., 2006; Peuker, Fogaça \& Bizarro, 2006; Pillon \& Corradi-Webster, 2006; Silva, Malbergier, Stempliuk \& Andrade, 2006).

Enfatizar tal preocupação é contribuir para a existência de fatores de proteção social e psicológica; esta reflexão, tem como base o estudo de Formiga, Picanço, et. al. (2013), pois, não basta apenas avaliar problema em termos de padrão de conduta, mas, também, variáveis psicológicas ou psicossociais que possam influenciar este fenômeno; de acordo com os autores supracitados, quando compararam o consumo de álcool por parte dos universitários e pessoas em vulnerabilidades (prostitutas, moradores de ruas, etc.), observaram diferença muito pequena na percentagem de respostas entre tais grupos em relação ao 
padrão consumo de álcool, sinais sintoma de dependência e problemas uso álcool. Com isso, estando os universitários em zona de risco grave quanto ao consumo de álcool, qual e como estes estariam dispostos para estudar e terem êxito acadêmico e profissional. Um estudo que tem direção semelhante aos resultados observados na presente pesquisa, foi desenvolvido por Formiga, Galdino, Ribeiro e Souza (2013); estes autores, além observarem uma consistência correlacional itens fatores tanto entre a pontuação total do AUDIT quanto com os respectivos fatores, pois tinham o objetivo de verificar a representatividade comportamento-domínio, também observaram uma consistência dessa medida, com base nos alfas de Cronbach; porém, apesar de resultados confiáveis, o estudo desses autores é limitado em relação ao resultado desta pesquisa, pois não abordaram análises multi-amostral e com distintas estatísticas.

Com base nestes resultados busca-se não apenas salientar a consistência de uma medida psicológica e epidêmica sobre o consumo de álcool, mas que é importante avaliar tanto a entrada e saída do universitário na instituição e sua socialização no consumo desta substância, a fim de promover políticas e projetos educacionais que devem ir além do processo ensino-aprendizagem e relação professor-aluno, mas que contemple uma formação de educação social sobre o problema com o álcool, gerando fatores de proteção na saúde física e mental dos estudantes. Ao salientar tais perspectivas conceituais na convergência estatística-teoria, não se trata, simplesmente, de uma melhoria psicométrica na especificidade e indexação entre os itens e seus respectivos fatores quanto ao tipo de estatística; busca-se oferecer indicadores mais confiáveis para a mensuração do construto em questão quando se pretender avaliar um fenômeno de alta complexidade e gravidade no ser humano (especialmente, nos universitários) e que sugere aplicações nos mais variados espaços das relações humanas e sociais.

\section{CONSIDERAÇÕES FINAIS}

Espera-se que o objetivo deste estudo tenha sido cumprido, principalmente, no que diz respeito à consistência e fidedignidade da estrutura fatorial do instrumento AUDIT; considerando os achados neste estudo, acredita-se que eles seriam úteis no emprego em áreas afins da psicologia, por exemplo: educação, assistência social, saúde, etc., pois, cada uma dessas áreas, em sua 
especificidade teórica e interventiva, contribuiria para uma maior atuação no controle e conscientização do problema no contexto universitário. Todavia, mesmo que esses resultados sejam confiáveis, destaca-se a necessidade de replicação dos mesmos em outros contextos sociais, políticos, educacionais e econômicos, bem como, considerar os aspectos mais específicos ou universais de cada cultura.

Por um lado, é importante considerar as dimensões locais, específicas ou exclusivas (emics) da orientação de cada cultura, bem como, e não menos importante, avaliar as dimensões universais (etics) da Cultura, com o objetivo de comparar os construtos estudados aqui para outro espaço geo-político e social (Triandis et. al., 1993; Triandis, 1996; Van De Vijve \& Leung, 1997; Muenjohn \& Armstrong, 2007). Apesar desses resultados comprovarem o que se hipotetizava, alguns limites devem ser considerados para futuros estudos: seria útil conhecer os aspectos que podem ser comuns em universitários de outros Estados brasileiros, bem como aqueles que são específicos, contribuindo assim, para consolidar um marco teórico da teoria e mensuração do problema com o álcool; outro estudo que contribuiria para o tema em questão trata-se de focar na avaliação das variações das respostas nestes construtos a partir da socialização de pares de iguais na universidade, tipo de lazer ou diversão, etc; também seria importante, reunir evidências da validade e precisão intra, inter e pan-cultural, de validade convergente com construtos correlatos, bem como, conhecer a estabilidade temporal (teste-reteste) replicando o mesmo instrumento com amostras maiores e mais diversificadas quanto às características dos participantes.

\section{REFERÊNCIAS}

Agante, D. M. C. (2009). Comportamentos relacionados com o consumo de bebidas alcoólicas durante as festas académicas nos estudantes do ensino superior. (Dissertação de Mestrado não publicada) - Faculdade de Medicina, Universidade de Coimbra, Portugal.

American Psychiatric Association. (1994). Diagnostic and statistical manual of mental disorders. 4th Ed. Washington DC: American Psychiatric Association.

Andrade, A. G., Anthony, J. C. \&, Silveira, C. M. (2009). Álcool e suas consequências: uma abordagem multiconceitual. Barueri, SP: Minha Editora. 
Associação Nacional de Pesquisa e Pós-Graduação em Psicologia. (2000). Contribuições para a discussão das Resoluções CNS no. 196/96 e CFP No 016/2000. Disponível em: http://www.anpepp.org.br/XIISimposio/Rel_ComissaoEticasobre_Res_CNS_ e_CFP.pdf.

Babor, T. F. et al. (1992). O alcohol use disorders identification test: orientações para o uso em saúde. Genebra: OMS.

Babor, T. F., Higgins-Biddle, J. C., Saunders, J. B., \& Monteiro, M. G. (2001). Audit: the alcohol use desorders identification test. Guidelines for Use in Primary Care. WHO/PSA.

Babor, T. F., \& Higgins-Biddle, J. C. (2001). Brief Intervention. For Hazardous and Harmful Drinking. A Manual for Use in Primary Care. WHO/PSA.

Bergman, H., \& Källmén, H. (2002). Alcohol use among Swedes and a psychometric evaluation of the Alcohol Use Disorder Identification Test. Alcohol, 37, 245-251.

Breda, J. J. R. S. (2010). Problemas ligados ao álcool em Portugal: Contributos para uma estratégia compreensiva. (Tese de Doutorado não publicada) Faculdade de Ciências da Nutrição e Alimentação, Universidade do Porto, Portugal.

Bye, E. K. \& Rossow, I. (2010). The impact of drinking pattern on alcohol related violence among adolescents: An international comparative analysis. Drug and Alcohol Review, 29(2), 131-136.

Byrne, B. M. (1989). A primer of LISREL: Basic applications and programming for confirmatory factor analytic models. New York: Springer-Verlag.

Carlini, E. A., Carlini-Contrim, B., \& Silva-Filho, A. R. (2007). II levantamento nacional sobre o uso de psicotrópicas em estudantes de $1^{\circ}$ e $2^{\circ}$ graus. São Paulo: Centro de Informações sobre Drogas Psicotrópicas, Departamento de Psicobiologia da Universidade Federal de São Paulo.

Carlini-Cotrim, B., Gazal-Carvalho, C., \& Gouveia, N. (2000). Comportamento de saúde entre jovens estudantes das redes pública e privada da área metropolitana do estado de São Paulo. Revista de Saúde Pública, 34(6), 636-345. 
Carvalho, F. N. (2010). Hábitos alcoólicos dos estudantes do mestrado integrado em Medicina da Universidade da Beira Interior (Dissertação de Mestrado não publicada). Programa de Pós-Graduação em Ciências da Saúde, Universidade da Beira Interior, Portugal.

Chalub, M., \& Telles, L. E. B. (2006). Álcool, drogas e crime. Revista Brasileira de Psiquiatria, 28, 69-73.

Conselho Nacional De Saúde. (2012). Diretrizes e normas regulamentadoras de pesquisas envolvendo seres humanos. Disponível em: http://conselho.saude.gov.br/resolucoes/reso_96.htm.

Dallo, L., \& Martins, R. A. (2011). Uso de álcool entre adolescentes escolares: Um estudo-piloto. Paidéia (Ribeirão Preto), 21(50), 329-334.

Fachini, A. (2009). Influência de expectativas e do grupo de pares sobre o comportamento do uso de álcool entre estudantes da área da saúde: uma perspectiva das diferenças de gênero. (Dissertação de Mestrado não publicada). Faculdade de Medicina de Ribeirão Preto, Universidade de São Paulo.

Figlie, N. B., Pillon, S. C., Laranjeira, R. R., \& Dunn, J. (1997). Audit indentifica a necessidade de interconsulta específica para dependentes de álcool no hospital geral? Jornal Brasileiro de Psiquiatria, 46(11), 589-593.

Formiga, N. S. (2011). Um nexo causal entre variáveis da violência em jovens. Caderno de Pesquisa Interdisciplinar em Ciências Humanas, 12(100), 86104.

Formiga, N. S., Galdino, R. M. G. M., Ribeiro, K. G. O. \&, Souza, R. C. (2013). Identificação de problemas relacionados ao uso de álcool (AUDIT): A fidedignidade de uma medida sobre o consumo exagerado de álcool em universitários. Psicologia.com.pt, 1, 1-13, Disponível em: http://www.psicologia.pt/

Formiga, N. S., Picanço, E. L., Souza, R. C. M., \& Santos, J. D. B. (2013). Identificação do problema com o consumo álcoolico em pessoas vulneráveis e não vulneráveis e sua relação com autoestima. Psicologia.com.pt, 1, 1-18. Disponível em: http://www.psicologia.pt/

Garcia, N. A. A., Aguilar, L. R., \& Facundo, F. R. G. (2008). Efecto de la autoestima sobre el consumo de tabaco y alcohol en adolescentes del área rural de Nuevo León, México. SMAD, Revista Electrónica en Salud Mental, Alcohol y Drogas, 4(1), 1-17. 
Hair, J. F., Tatham, R. L., Anderson, R. E., \& Black, W. (2005). Análise multivariada de dados. Porto Alegre: Bookman.

Joreskög, K., \& Sörbom, D. (1989). LISREL 7 user's reference guide. Mooresville: Scientific Software.

Kelloway, E. K. (1998). Using LISREL for structural equation modeling: $A$ researcher's guide. Thousand Oaks, CA: Sage Publications.

Kerr-Corrêa, F., Dalben, I., Trinca, L. A., Simão, M. O., Mattos, P. F., RamosCerqueira, A. T. A., \& Mendes, A. A. (Eds.). (2001). I Levantamento do uso de álcool e de drogas e das condições gerais de vida dos estudantes da UNESP (1998), (Vol. 14). Pesquisa V. Unesp. São Paulo, SP: Fundação para o Vestibular da Universidade Estadual Paulista.

Kerr-Corrêa, F., Simão, M. O., Dalben, I., Martins, R. A., Trinca, L. A., Penteado, M. A. C., Sanches, A. F., Oliveira, S. M., Beig, M. L., \& Ortigosa, S. (2002, Julho). High risk alcohol use in Brazilian college students (UNESP): Preliminary data from a preventive study. Full Papers presented of the XXVIII Annual Alcohol Epidemiology Symposium, Paris.

Laranjeira, R., Pinsky, I., Zaleski, M., \& Caetano, R. (2007). I Levantamento Nacional sobre os Padrões de Consumo de Álcool na População Brasileira. Brasília, DF: Secretaria Nacional de Políticas sobre Drogas.

Llorens A., Palmer, P., \& Perellón del Río, M. (2005). Características de personalidad en adolescentes como predictores de la conducta de consum 0 de sustancias psicoactivas. Transtornos Adictivos, 7(2), 90-96.

Lucas, A. C. S. et al. (2006). Uso de psicotrópicos entre universitários da área da saúde da Universidade Federal do Amazonas, Brasil. Caderno de Saúde Pública, 22(3), 663-667.

Margotto, P. R. (2010). Curva ROC como fazer e interpretar no SPSS. Disponível em: http://www.paulomargotto.com.br/documentos/Curva_ROC_SPSS.pdf

Martins, J. R. S. V. (2009). O consumo de bebidas alcoólicas nos adolescentes. (Monografia de licenciatura em ciências da enfermagem). Porto, Portugal. Universidade Fernando Pessoa.

Moretti-Pires, R. O., \& Corradi-Webster, C. M. (2011). Adaptação e Validação do Álcohol use Disorder Identification Test (AUDIT) para a população ribeirinha do interior da Amazônia, Brasil. Cadernos de Saúde Pública, 27(3), 497509. 
Muenjohn, N. \&, Armstrong, A. (2007). Transformational leadership: The influence of culture on the leadership nehaviours of expatriate managers. International Journal of Business and Information, 2(2), 265-283.

Musitu, G., Jiménez, T. I., \& Murgui, S. (2007). Funcionamiento familiar, autoestima y consumo de sustancias en adolescentes: Un modelo de mediación. Salud Pública México, 49(1), 3-10.

Navarro, H., \& Pontillo, C. H. (2002). Autoestima del adolescente y riesgo de consumo de alcohol. Actualización en Enfermería, 5(1), 7-12.

Oliveira, J. C., \& Andrade, F. W. M. (2002). Comparação entre medidas de performance de modelos de credit scoring. Tecnologia de Crédito, 33, 3547.

Pastor, Y., Balaguer, I., \& García-Merita, M. L. (2000). Estilo de vida saludable en la adolescencia media: análisis diferencial por curso y sexo. Revista de Psicología de la Salud, 12(2), 55-74.

Peuker, A. C., Fogaça, J., \& Bizarro, L. (2006). Expectativas e beber problemático entre universitários. Psicologia: Teoria e Pesquisa, 22(2), 193200.

Pillon, S. C., \& Corradi-Webster, C. M. (2006). Teste de identificação de problemas relacionados ao uso de álcool entre estudantes universitários. Revista de Enfermagem, 14, 325-332.

Romera, L. A. (2008). Juventude, lazer e uso abusivo de álcool. Disponível em: http:// www. Bibliotecadigital.unicamp.br/document/code=vtls000445959.

Saunders, J. B., Aasland, O. G., Babor, T. F., de la Fuente, J. R., \& Grant, M. (1993). Development of the Alcohol Use Disorders Screening Test (AUDIT). WHO collaborative project on early detection of persons with harmful alcohol consumption. II. Addiction, 88, 791-804.

Silva, L. V. E. R., Malbergier, A., Stempliuk, V. A., \& Andrade, A. G. (2006). Fatores associados ao consumo de álcool e drogas entre estudantes universitários. Revista de Saúde Pública, 40(2), 280-288.

Triandis, H. C. (1996). The psychological measurement of cultural syndromes. American Psychologist, 51, 407-415.

Triandis, H. C., McCusker, C., Betancourt, H., Iwao, S., Leung, K., Salazar, J. M., Setiadi, B., Sinha, B. P., Touzard, H., \& Zaleski, Z. (1993). An etic-emic analysis of individualism and collectivism. Journal of Cross-cultural Psychology, 24(3), 366-383. 
Van de Vijver, F., \&, Leung, K., (1997). Methods and data analysis for crosscultural research. Thousand Oaks, CA: Sage Publications.

World Health Organization (2004). Global status report on alcohol 2004. Geneva: World Health Organization.

Contato: nsformiga@yahoo.com

Recebido em: 31/12/2013

Revisado em: 20/02/2014

Aceito em: 26/02/2014 